Prise en charge chirurgicale d'une rétention trophoblastique après grossesse sur cicatrice de césarienne et réparation d'une isthmocèle par colioscopie robot-assistée associée à l'hystéroscopie.

Robotic-assisted laparoscopy using hysteroscopy treatment of a residual cesarean scar pregnancy and isthmocele.

Titre court : cure chirurgical d'isthmocèle

Krystel NYANGOH TIMOH, MD, PHD, Isabelle ENDERLE, MD, Vincent LAVOUE, MD, PHD.

${ }^{1}$ Department of Obstetrics and Gynecology, Rennes Hospital. Rennes University 1, Rennes, France.

${ }^{2}$ Université de Rennes $1: 2$ avenue de Professeur Léon Bernard, 35000

3 Equipe thématique SAFE, CIC 1414

Correspondance :

Docteur Krystel Nyangoh Timoh.

Service de Gynécologie-Obstétrique. Hôpital Sud Rennes. Rennes. France.

Tel: +3372136050

Email: krystel.NYANGOH.TIMOH@chu-rennes.fr

Conflit d'intérêt : aucun

Prise en charge chirurgicale d'une rétention trophoblastique après grossesse sur cicatrice de césarienne et réparation d'une isthmocèle par colioscopie robot-assistée associée à l'hystéroscopie.

Robotic-assisted laparoscopy using hysteroscopy treatment of a residual cesarean scar pregnancy and isthmocele.

Krystel NYANGOH TIMOH, MD, PHD, Isabelle ENDERLE, MD, Jean LEVEQUE, MD, PHD, Vincent LAVOUE, MD, PHD.

${ }^{1}$ Department of Obstetrics and Gynecology, Rennes Hospital. Rennes University 1, Rennes, France.

${ }^{2}$ Université de Rennes $1: 2$ avenue de Professeur Léon Bernard, 35000

3. Equipe thématique SAFE, CIC 1414

Correspondance :

Docteur Nyangoh Timoh Krystel.

Service de Gynécologie-Obstétrique. Hôpital Sud Rennes. Rennes. France.

Tel: +3372136050

Email: krystel.NYANGOH.TIMOH@chu-rennes.fr

Conflit d'intérêt : aucun 


\section{Objectif :}

Décrire la technique chirurgicale de prise en charge d'une isthmocèle et d'une rétention trophoblastique de grossesse sur cicatrice de césarienne.

Méthodes :

Une patiente de 38 ans a consulté pour aménorrhée depuis 5 mois après une fausse couche spontanée. Elle avait deux antécédents de césarienne.

Une échographie pelvienne et une IRM pelvienne montraient une rétention trophoblastique au sein d'un isthmocèle. Le mur myométrial résiduel était de $1 \mathrm{~mm}$. La hauteur de l'isthmocèle était de $15 \mathrm{~mm}$.

Dans cette vidéo, nous avons décrit la technique d'évacuation de la rétention trophoblastique sur isthmocèle et de réparation de du défect sur cicatrice de césarienne utilisant la cœlioscopie robot-assistée et l'hystéroscopie.

La première étape était le clivage vésico-utérin permettant la libération de la vessie de ses adhérences avec l'isthmocèle. Ensuite l'hystéroscopie était réalisée. La lumière de l'optique de coelioscopie était diminuée. Et Enfin on excisait le tissu cicatriciel autour de l'isthmocèle pour atteindre le tissu sain. Les parois de l'utérus étaient alors suturées par des points séparés au fil d'absorption lente.

\section{Résultat}

Le temps opératoire était de $120 \mathrm{~min}$. Aucune complication per et post-opératoire n'ont été rapportées. La patiente a été hospitalisée $48 \mathrm{~h}$.

\section{Conclusion}

La cœlioscopie robot-assistée utilisant l'hystéroscope pour la prise en charge des isthmocèles et d'une rétention trophoblastique sur grossesse sur cicatrice de césarienne est une procédure simple et faisable.

Mots clés : isthmocèle; défect de la cicatrice de césarienne ; grossesse sur cicatrice de césarienne ; complication de césarienne ; cœlioscopie ; robot

Keywords : Isthmocele; cesarean section scar defect; cesarean section scar pregnancy; cesarean section complication; laparoscopy; robot

Les auteurs remercient Christophe Morvan ( Laboratoire Audiovisuel, CHU Rennes) 


\section{Références}

1. Akdemir A, Sahin C, Ari SA, Ergenoglu M, Ulukus M, Karakadas N. Determination of Isthmocele Using a Foley Catheter During Laparoscopic Repair of Cesarean Scar Defect. J Minim Invasive Gynecol 2018;25:21-22

2. Api M, Boza A, Gorgen H, Api O. Should Cesarean Scar Defect Be Treated Laparoscopically? A Case Report and Review of the Literature. J Minim Invasive Gynecol 2015;22:1145-52.

3. Donnez O, Donnez J, Orellana R, Dolmans MM. Gynecological and obstetrical outcomes after laparoscopic repair of a cesarean scar defect in a series of 38 women. Fertil Steril 2017;107:289-96 e2. 